\title{
Low-Temperature Reverse Microemulsion Synthesis, Characterization, and Photocatalytic Performance of Nanocrystalline Titanium Dioxide
}

\author{
Zhang Liu, ${ }^{1}$ Zicong Jian, ${ }^{2}$ Jianzhang Fang, ${ }^{1}$ Xiaoxin $\mathrm{Xu},{ }^{1}$ Ximiao Zhu, ${ }^{1}$ and Shuxing Wu${ }^{1}$ \\ ${ }^{1}$ School of Chemistry and Environment, South China Normal University, Guangzhou 510006, China \\ ${ }^{2}$ School of Environment, South China University of Technology, Guangzhou 510640, China \\ Correspondence should be addressed to Jianzhang Fang, fangjzh@scnu.edu.cn
}

Received 30 October 2011; Revised 24 November 2011; Accepted 24 November 2011

Academic Editor: Jiaguo Yu

Copyright () 2012 Zhang Liu et al. This is an open access article distributed under the Creative Commons Attribution License, which permits unrestricted use, distribution, and reproduction in any medium, provided the original work is properly cited.

\begin{abstract}
Nanocrystalline titanium dioxide $\left(\mathrm{TiO}_{2}\right)$ was synthesized in microemulsions by using cetyltrimethylammonium bromide (CTAB) as surfactant. In order to investigate the crystal transformation and photoactivity at low temperature, the as-prepared precipitates were aged at $65^{\circ} \mathrm{C}$ or calcined at various temperatures. Analyses using powder X-ray diffraction (XRD) and Fourier transform infrared microscopy (FT-IR) showed that precursors without aging or calcination were noncrystal and adsorbed by surfactant. After aging for $6 \mathrm{~h}$, the amorphous $\mathrm{TiO}_{2}$ began to change into anatase. The obtained catalysts, which were synthesized in microemulsions with weight ratios of n-hexanol/CTAB/water as $6: 3: 1$ and calcined at $500^{\circ} \mathrm{C}$, presented the highest photocatalytic degradation rate on methyl orange (MO), while the catalysts, which were aged at $65^{\circ} \mathrm{C}$ for $90 \mathrm{~h}$, also exhibited an outstanding photocatalytic performance and a little higher than that of the commercial titania photocatalyst Degussa P25.
\end{abstract}

\section{Introduction}

$\mathrm{TiO}_{2}$ is well-known for its applications in oxide semiconductors, oxygen sensors, photovoltaics, photocatalysis, and pigments [1-4]. Extensive researches have been focus on synthetic methods of semiconductor nanoparticles with better crystallinity and photocatalytic performance $[5,6]$. Among various approaches explored, the reverse microemulsion process seems an efficient way to obtain nanosized $\mathrm{TiO}_{2}$ [7]. Many scholars $[8,9]$ suggested that the main advantages of the microemulsion method were the low reaction temperature, the short processing time, and the attractive effect of preventing agglomeration in the formed nanoparticles. The property of microemulsion is mainly determined by the characteristics of the forming surfactant. Therefore, a proper surfactant may simplify producing the desired nanosized $\mathrm{TiO}_{2}$. One of the goals of this article is showing the application of microemulsions which acted as nanoparticles reactors.

In a typical procedure for $\mathrm{TiO}_{2}$ preparation, samples usually need to be treated under several hundred degrees centi- grade [10-12]. Some researches, however, have sought the possibility of synthesizing $\mathrm{TiO}_{2}$ in low temperatures, and obtained certain progress. Qi et al. [13] synthesized $\mathrm{TiO}_{2}$ at $100^{\circ} \mathrm{C}$ and discussed the heterogeneous nucleation mechanism. Liu et al. [14] obtained $\mathrm{TiO}_{2}$ with small crystalline size and large specific surface area around $100^{\circ} \mathrm{C}$. Although $\mathrm{TiO}_{2}$ obtained under low temperatures has been studied, the microemulsion-resulted products which underwent different aging time and related photocatalytic activities were seldom discussed.

In the present study, the phase behavior of cetyltrimethylammonium bromide (CTAB) in $n$-hexanol/water system was studied through the electrical conductivity and the phase diagrams. Nanosized $\mathrm{TiO}_{2}$ particles were synthesized in $n$-hexanol/CTAB/water reverse microemulsion under low aging temperature or high calcination temperature, and the powders were characterized by X-ray diffraction (XRD), Fourier transform infrared spectroscopy (FT-IR), and transmission electron microscopy (TEM) techniques. A simulated organic pollutant, methyl orange (MO), was decomposed on $\mathrm{TiO}_{2}$ suspensions under UV irradiation. 


\section{Materials and Methods}

2.1. Analysis of Microemulsions. The electrical conductivity of ternary system was measured using a DDS-307 conductivity meter (Leici Instruments, Shang Hai, China). Deionized water was added dropwise to the mixture of n-hexanol and CTAB at room temperature until the system became turbid. The weight ratio of $n$-hexanol to CTAB was fixed at 2, while the water concentration was represented by quality percentage.

Depending on the preparation procedure of microemulsions, phase diagrams were constructed using a conventional titration technique and the homogeneous phase regions were obtained at different temperatures $\left(25,65,80^{\circ} \mathrm{C}\right)$. Stock solutions of CTAB and n-hexanol at the certain weight ratio were prepared at room temperature. Deionized water was added dropwise to glass vials containing n-hexanol/CTAB mixtures, which were maintained at various temperatures in water bath. The as-prepared microemulsions were sealed and shaken by a magnetic stirrer. The mass of added water was determined by weighting the glass vial before and after adding to the mixture.

2.2. Preparation of $\mathrm{TiO}_{2}$ Nanoparticles. In a typical procedure, microemulsion of $\mathrm{n}$-hexanol:CTAB: water with weight ratio of $6: 3: 1$, was firstly prepared by mixing three component through a magnetic stirrer and stirred for $60 \mathrm{~min}$ until the systems became clear and equality. Maintaining that the molar ratio of water to $\mathrm{Ti}(\mathrm{OBu})_{4}$ is 15 , a specific weight of $\mathrm{Ti}(\mathrm{OBu})_{4}$ was added dropwise into the microemulsion system and further stirred for $120 \mathrm{~min}$.

To obtain samples treated by high temperature, the solid precipitates were centrifuged from the microemulsion system and washed with ethanol and deionized water for five times. The precursors were dried at $105^{\circ} \mathrm{C}$ for $12 \mathrm{~h}$. Then the sample was calcined for $3 \mathrm{~h}$ at $300,400,500,600$, and $700^{\circ} \mathrm{C}$.

To obtain samples treated by low temperature, after $\mathrm{Ti}(\mathrm{OBu})_{4}$ was added into the microemulsion and stirred for $120 \mathrm{~min}$, the whole mixtures were kept in water bath $\left(65^{\circ} \mathrm{C}\right)$ with different aging time. The final products were obtained after centrifugation, washing, and drying processes as above.

2.3. Photocatalytic Activity Measurement. The photocatalytic reaction was conducted in a $200 \mathrm{~mL}$ cylindrical glass vessel fixed in the XPA photochemical reactor (Nanjing Xujiang Machine-electronic Plant, China). The reactor consisted of magnetic stirrer, quartz cool trap, and condenser that can keep the reaction temperature steady and prevent the evaporation of water. A $300 \mathrm{~W} \mathrm{Hg}$ lamp with center wavelength of $365 \mathrm{~nm}$ was used as the UV light source. Methyl orange (MO) was used as a target pollutant, with an initial concentration of $10 \mathrm{mg} \mathrm{L}^{-1}$.

$100 \mathrm{mg}$ photocatalyst powder was dispersed in $200 \mathrm{~mL}$ MO solution sufficiently for $30 \mathrm{~min}$ to achieve adsorption/desorption equilibration. Subsequently, the $\mathrm{Hg}$ lamp was turn on. At regular intervals $(10 \mathrm{~min})$, about $5 \mathrm{~mL}$ of the suspension was sampled and filtered through a $0.45 \mu \mathrm{m}$

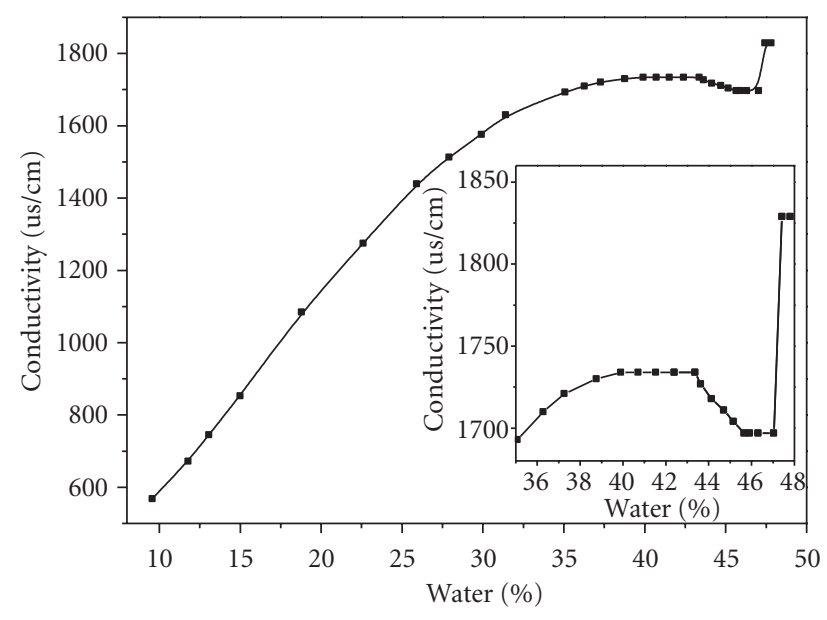

FIGURE 1: Relationship between the electrical conductivity and the water concentration with the weight ratio of $n$-hexanol to CTAB is 1.5 .

membrane filter. The concentration of the remaining pollutant was measured by its absorbance $(A)$ at $464 \mathrm{~nm}$ with a Hitachi UV-3010 spectrophotometer (Tokyo, Japan). The degradation ratio $(X)$ of the reactant was calculated by $X(\%)=100\left(A_{0}-A_{t}\right) / A_{0}$, where $A_{0}$ and $A_{t}$ are the absorbencies at time zero and at time $t$.

2.4. Characterization of $\mathrm{TiO}_{2}$ Nanoparticles. XRD patterns were recorded on a Dandong Aolong/Y-2000 X-ray diffractometer (Dan dong, China) with $\mathrm{Cu} K \alpha$ radiation $(\lambda=$ $0.15406 \mathrm{~nm})$. The average particle size was calculated according to the Scherrer equation $d=k \lambda / \beta \cos \theta$, where $k$ is a constant, $\lambda$ is the wavelength of the X-rays, $\theta$ is the angle at the maximum peak, and $\beta$ is the full width of the peak at half height. FT-IR spectra were recorded using a Shimadzu IRPrestige-21 Fourier transform spectrometer (Japan) by blending the sample into a $\mathrm{KBr}$ pellet. The particle size and morphology were observed on a JEOL JEM-2010 (HR) transmission electron microscope (Japan) (working voltage at $100 \mathrm{kv}$ ). The thermogravimetric analysis of precursors was measured using a STA449c/1/41G thermal analyzer (Netzsch, Germany).

\section{Results and Discussion}

3.1. Analysis of Microemulsions. In the ternary system, the electric conductivity is determined by the movement of counter-ions in water phase, and the oil phase was not conducting, which was confirmed by the conductivity measurement of $\mathrm{n}$-hexanol $\left(0.04 \mu \mathrm{s} \mathrm{cm}^{-1}\right)$. Bromine anions dissociated from the surfactant into the water phase while adding water, and this improved the conductive capacity when the anions pass through the membranes between reverse micelles during collision. Therefore, the diameter and the collision frequency of droplets are the main determinants for electrical conductivity. Figure 1 shows the conductivity of the n-hexanol/CTAB system related to water concentration. 
As it can be seen, conductivity increased rapidly when water concentration was raised from $10 \%$ to $35 \%$, and the mixture kept transparent. This was a solubilization process of reverse micelles. Water molecules gathered around the hydrophilic groups and formed water droplets. Adding water not only caused an increased amount of droplets, but also enlarged volumes of droplets, both of them resulted in the increase of collision frequency. According to the theory of sticky droplet collisions [15], many narrow water channels were formed in continuous phase when droplets collided. Hence, ions in water phase were able to pass through the oil phase via such channels and led to the increase of electrical conductivity. Moreover, adding water caused the ionization of CTAB, and this led to sharply increased amount of bromine anions. In a word, the conductivity presented nearly a straight increase with the adding water.

As water concentration rising from $35 \%$ to $43 \%$, conductivity increased slowly and reached a maximum; meanwhile, the mixture became viscous and exhibited a characteristic refraction which was an indicative of formation of liquid crystal. In this process, the volumes of water droplets were enlarged. Because the ionization of the surfactant molecules tended to be equilibrium [16], the amount of dissociative ions slowly increased to a maximum. In addition, the bigger droplets the lower migration rate, and this also reduced the conductivity. Once the liquid crystal formed, the interfacial film became rigid and the formation of conductance chains stopped. Thus, the electrical conductivity maintained at a certain value after a slow increase.

After the maximum stage, the conductivity reduced gradually to a minimum value as water concentration rise from $43 \%$ to $47 \%$. The system became ropier and appeared as opalescence. The numbers of dissociating ions kept unchanged as adding water, while the volume of droplets were enlarged so much that reduced their mobility. Moreover, when the system reaches the utmost of water content with lower migration, the interface arranged in no regulation. Hence, the viscosity collision replaced the elastic collision. The lower migration rate of water droplets would certainly result in the reduced electrical conductivity.

With the continuous addition of water, the electrical conductivity increased abruptly and the whole solution became opaque and turbid, and the system became multiphase. Such turbidity is called demulsification. Before demulsification, the mixture maintained monophase.

The water concentrations at the points of demulsification within certain weight ratios of n-hexanol to surfactant were recorded as the maximum concentration, while the ones at the points of mutual soluble of these three contents were recorded as the minimum concentration. The relation between water concentration and the weight ratios of n-hexanol to surfactant under different temperatures was shown in Figure 2.

As shown in Figure 2, solid lines represent the maximum concentration of water, while dashed lines represents the minimum, and the region between two lines is the monophase region of the $\mathrm{n}$-hexanol/CTAB/water system. The maximum water concentration increased sharply and reached a high value as the ratio increased to 1.6, and fell back

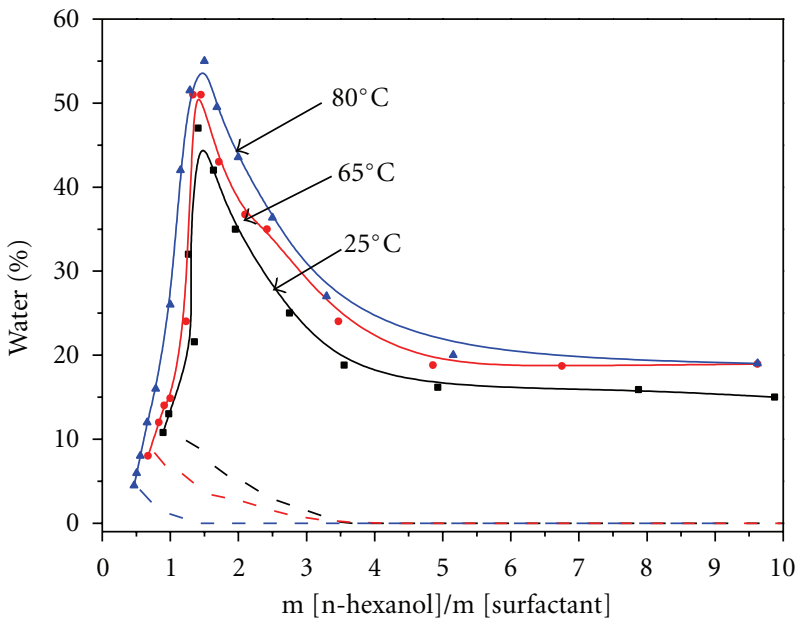

FIGURE 2: Relationship between water solubilization and mass ratio of n-hexanol to CTAB.

to a stable value. This phenomenon of self-emulsification was due to that $n$-hexanol reacted as a cosurfactant and the oil phase at the same time. When the surfactant was more than n-hexanol, n-hexanol preformed as the cosurfactant that stabilized the interface layer and increased the water solubilization. When the weight ratio tended to 1.6, numbers of n-hexanol molecular in the interface layer were near saturation, and the excess n-hexanol existed as an oil phase which prevented the solubilization of water. Hence, the maximum water concentration was no longer determined only by nhexanol but also by the property of CTAB.

The area of the monophase region is affected obviously by temperature, which can be explained as follows high temperature can improve the hydrophilicity of surfactant, raise the number of water molecules that were controlled by each surfactant molecule, and enlarge the volume of droplets.

3.2. Characterization of Photocatalyst. In this work, the precursor was prepared in a reverse microemulsion of n-hexanol, $\mathrm{CTAB}$, and water with weight ratio of $6: 3: 1$ under 120 min vigorous stirring, and the final products were obtained by further treatment of precursors by calcination and aging methods.

TG-DSC curve for precursor was shown in Figure 3. TG graph revealed that from 50 to $100^{\circ} \mathrm{C}$, the sample lost weight slowly, which was attributed to the volatilization of physically absorbed water. From 100 to $280^{\circ} \mathrm{C}$, the precursor had a gradual weight loss, which was caused by the evaporation of $\mathrm{n}$-hexanol (boiling point at $156^{\circ} \mathrm{C}$ ). As the temperature increased from 280 to $320^{\circ} \mathrm{C}$, the precursor had a sharp weight loss, which corresponded to the burning of residual surfactant. Such burning also caused a weak exothermic peak in DSC curve around $300^{\circ} \mathrm{C}$. After $320^{\circ} \mathrm{C}$, the curve was obtained as flat, indicating that the weight of precursor had little change.

DSC curve of precursor showed one endothermic peak at $120^{\circ} \mathrm{C}$ due to the evaporation of $\mathrm{n}$-hexanol. In the subsequent process, one main exothermic peak and two weak 


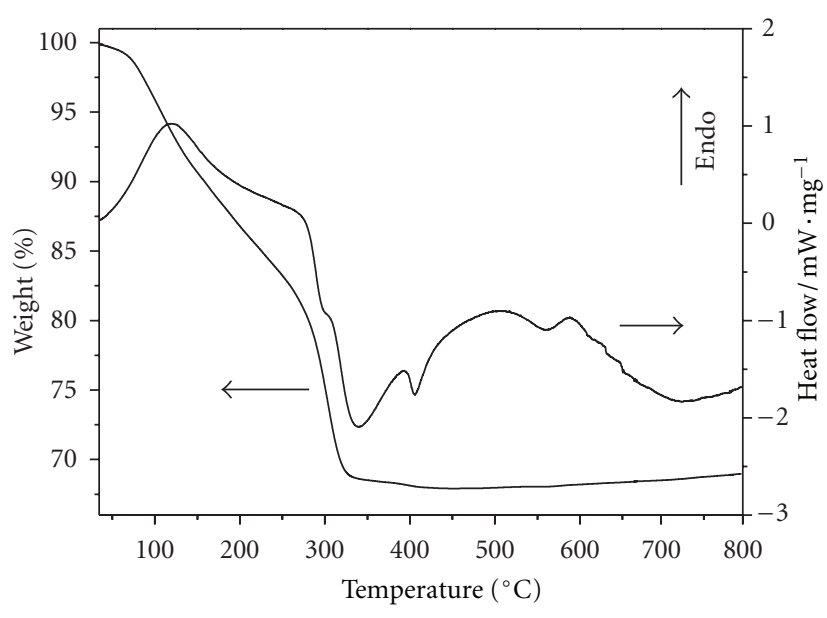

FIGURE 3: TG-DSC curves of a precursor.

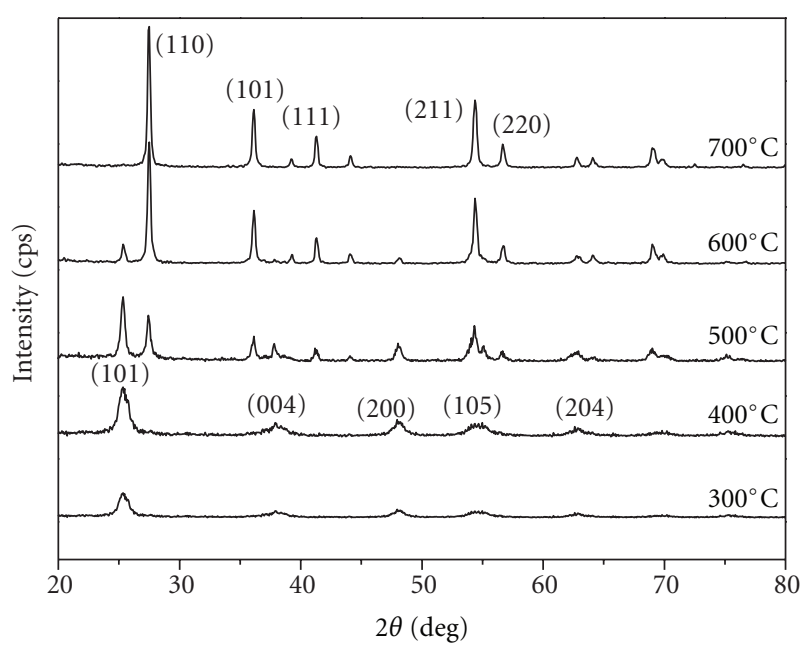

Figure 4: XRD patterns of $\mathrm{TiO}_{2}$ precursor calcined at different temperatures.

exothermic peaks were obtained. The first one around 300$320^{\circ} \mathrm{C}$ was caused by the burning surfactant which has been discussed above, the one at $400^{\circ} \mathrm{C}$ corresponded to the crystallization of anatase, and the third one at $500-600^{\circ} \mathrm{C}$ was the crystallization of rutile. These transitions of crystal phase could be confirmed by XRD pattern.

Figure 4 shows the XRD pattern of $\mathrm{TiO}_{2}$ calcined at different temperatures. At $400^{\circ} \mathrm{C}$ the XRD pattern showed a weak peak at $2 \theta=25.30^{\circ}$, implying that $\mathrm{TiO}_{2}$ was crystallized purely as anatase phase. The XRD patterns of samples calcined at 500 and $600^{\circ} \mathrm{C}$ showed peaks at $2 \theta=25.30^{\circ}$ and $2 \theta=27.50^{\circ}$, indicating that both anatase and rutile existed. No anatase peak was found in the XRD pattern of the sample calcined at $700^{\circ} \mathrm{C}$. Moreover, as the calcination temperature increased, all peaks became stronger and sharper, implying that the crystallinity became higher. Altogether, the XRD patterns presented the process of transition from anatase to rutile.
TABLE 1: Crystallite structure and size of $\mathrm{TiO}_{2}$ calcined at different temperature.

\begin{tabular}{lcc}
\hline Temperature $\left({ }^{\circ} \mathrm{C}\right)$ & Structure & Crystallite size $(\mathrm{nm})$ \\
\hline 300 & $100 \% \mathrm{~A}$ & $19 \mathrm{~A}$ \\
400 & $100 \% \mathrm{~A}$ & $20 \mathrm{~A}$ \\
500 & $61 \% \mathrm{~A}+39 \% \mathrm{R}$ & $25 \mathrm{~A}+21 \mathrm{R}$ \\
600 & $12 \% \mathrm{~A}+88 \% \mathrm{R}$ & $29 \mathrm{~A}+27 \mathrm{R}$ \\
700 & $3 \% \mathrm{~A}+97 \% \mathrm{R}$ & $33 \mathrm{R}$ \\
\hline
\end{tabular}

R: rutile; A: anatase.

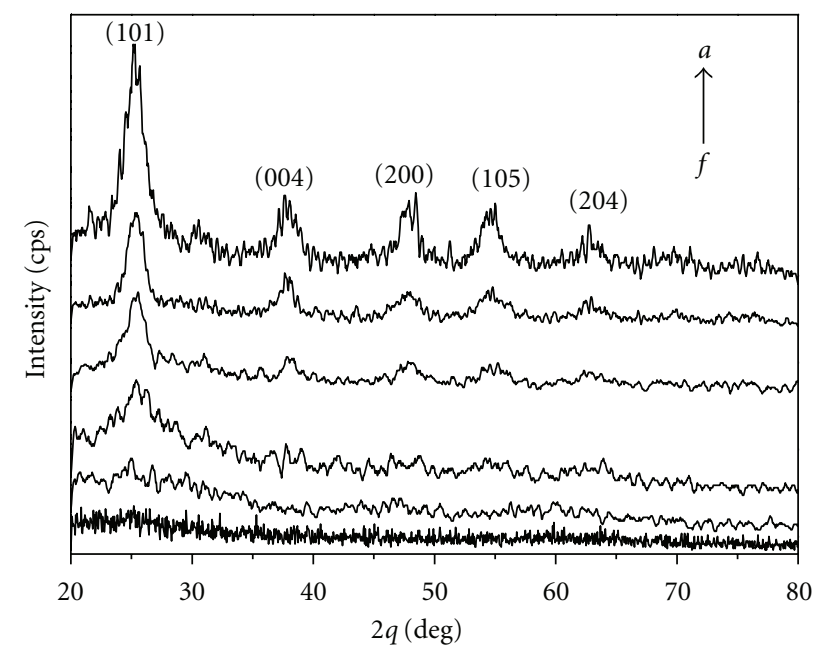

Figure 5: XRD patterns of $\mathrm{TiO}_{2}$ precursor aged at $65^{\circ} \mathrm{C}$ for (a) $0 \mathrm{~h}$, (b) $6 \mathrm{~h}$, (c) $12 \mathrm{~h}$, (d) $48 \mathrm{~h}$, (e) $90 \mathrm{~h}$, (f) $135 \mathrm{~h}$.

The average particle size, according to the Scherrer equation $d=k \lambda / \beta \cos \theta$, was shown in Table 1 . As the temperature raise, the average particle size rises from 19 to $33 \mathrm{~nm}$.

Figure 5 presents the XRD patterns of $\mathrm{TiO}_{2}$ aged at $65^{\circ} \mathrm{C}$ from $0 \mathrm{~h}$ to $135 \mathrm{~h}$. The precursor, that is, the sample aged for $0 \mathrm{~h}$, displayed amorphous phase with no peak in the XRD pattern. The sample represented a very weak peak at $2 \theta=25.30^{\circ}$ after aging for $6 \mathrm{~h}$, indicating $\mathrm{TiO}_{2}$ began to show the symptom of crystallization. As the aging proceeded, characteristic peaks grew slowly, and all these peaks indicated that the samples were crystallized as pure anatase. The crystallinity became better and better as the aging time became longer; however, the patterns showed little difference between samples aged for $48 \mathrm{~h}, 90 \mathrm{~h}$, or $135 \mathrm{~h}$. It was obviously that all the peaks were wide, which means the diameters of $\mathrm{TiO}_{2}$ crystal domain were small.

The diameters of the particles, according to the Scherrer equation, were shown in Table 2. As the aging time prolong, the average crystallite size rise from 0 to $4.8 \mathrm{~nm}$. Samples aged longer than $48 \mathrm{~h}$ were almost unchanged in crystallite size.

The mechanism of $\mathrm{TiO}_{2}$ crystal growth is considered as a process of diffusion controlled, and according to the phase transition theory of crystallization [17], relation between crystallite size of anatase and aging time could be fitted as

$$
d_{A}^{5}-d_{A 0}^{5}=k t^{f} \text {, }
$$


TABLE 2: Crystallite structure and size of $\mathrm{TiO}_{2}$ at different aging time.

\begin{tabular}{lcc}
\hline Ageing time $(\mathrm{h})$ & Structure & Crystallite size $(\mathrm{nm})$ \\
\hline 0 & Unformed & - \\
6 & Unformed & 0.99 \\
12 & Anatase & 1.33 \\
24 & Anatase & 3.66 \\
48 & Anatase & 4.22 \\
72 & Anatase & 4.42 \\
102 & Anatase & 4.55 \\
114 & Anatase & 4.63 \\
120 & Anatase & 4.73 \\
135 & Anatase & 4.83 \\
\hline
\end{tabular}

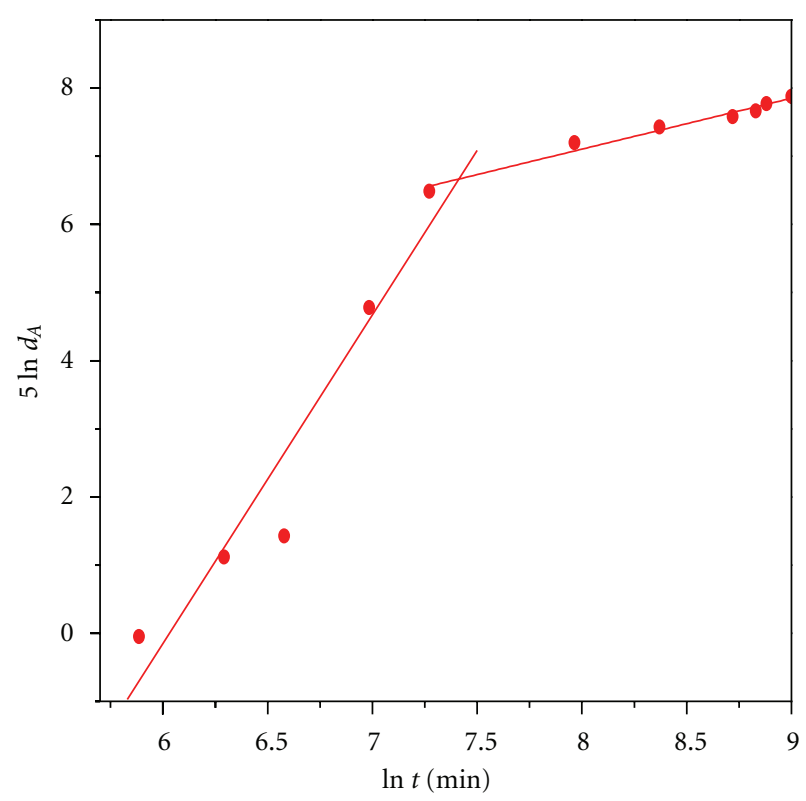

Figure 6: Fitting curves of $5 \ln d$ and $\ln t$.

where $d_{A 0}(\mathrm{~nm})$ is the initial diameter; $d_{A}(\mathrm{~nm})$ is the diameter when aging time is $t(\mathrm{~min}) ; f$ is the series of aging time to diameter grow; $k$ is the rate of crystal growth. Considering $d_{A}^{5} \gg d_{A 0}^{5}$, the natural logarithm of (1) is simplified as

$$
5 \ln d_{A}=f \ln t+\ln k .
$$

Equation (2) is the relation between aging time $(t / \mathrm{min})$ and crystallite size $\left(d_{A} / \mathrm{nm}\right)$. Took $\ln t$ as transverse axis and $5 \ln d_{A}$ as vertical axis, and constructed their linearity, the relation are shown in Figure 6. Fitting curves were obtained by subsection simulation. Table 3 showed the series of aging time to diameter grow $f$ and the rate of crystal growth $k$.

Fitting curves showed that, at the beginning of aging process, crystallite grew quickly with an extreme low rate of crystal growth. Such phenomenon might be explained by the phase transition from amorphous to anatase. During this stage, system formed plenty of crystal nucleuses. After being aged for $26 \mathrm{~h}$, the growth of crystal was not obvious, and
TABLE 3: Rate constant of crystal growth and chronological series.

\begin{tabular}{lccc}
\hline Stage & $f$ & $\ln k$ & $k$ \\
\hline 1 & 4.83169 & -29.15146 & $2.19 \times 10^{-13}$ \\
2 & 0.74674 & 1.13029 & 3.10 \\
$t_{a}$ & & $1588 / \min (26 / \mathrm{h})$ & \\
\hline
\end{tabular}

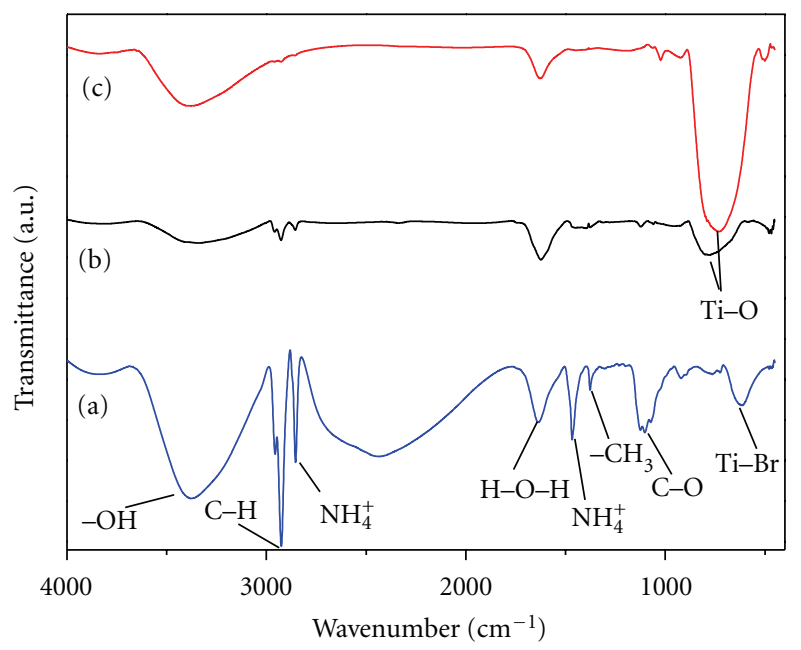

FIgURE 7: FT-IR spectra of (a) $\mathrm{TiO}_{2}$ precursor, (b) $\mathrm{TiO}_{2}$ precursor aging at $65^{\circ} \mathrm{C}$ for $120 \mathrm{~h},(\mathrm{c}) \mathrm{TiO}_{2}$ precursor calcined at $500^{\circ} \mathrm{C}$.

the diameter was effected slightly by aging time $(f=0.75)$. This might be the process that crystal grew from imperfect to perfect. The work showed that anatase could be obtained at low temperature.

Figure 7 shows the FT-IR spectra of $\mathrm{TiO}_{2}$ precursor treated by calcination and aging process. The band at about $3400 \mathrm{~cm}^{-1}$ was indexed to the stretching vibration of the $\mathrm{O}-\mathrm{H}$ bond and free water and the band at about $1631 \mathrm{~cm}^{-1}$ was attributed to the $\mathrm{O}-\mathrm{H}$ bending vibration of chemically adsorbed water [18]. Bands at 2700 and $1462 \mathrm{~cm}^{-1}$ were considered as the characteristic peaks of ammonium; $2850 \mathrm{~cm}^{-1}$ was the stretching vibration band of $\mathrm{C}-\mathrm{H}$ bond in methylene, while $1050 \mathrm{~cm}^{-1}$ was the vibration of $\mathrm{C}-\mathrm{O}$ bond [19]. These bands showed that precursor adsorbed a large number of organic compounds, provided by $n$-hexanol and CTAB. Calcination or aging were able to remove these organic compounds. The stretching vibration of $\mathrm{Ti}-\mathrm{Br}$ bond caused a wide band around $600 \mathrm{~cm}^{-1}$, while band around $800 \mathrm{~cm}^{-1}$ was the characteristic peak of Ti-O bond $[18,19]$. These bands showed that, precursors must be heated to form Ti-O bond.

Figure 8 shows the typical TEM images of $\mathrm{TiO}_{2}$ aged at $65^{\circ} \mathrm{C}$ for $120 \mathrm{~h} . \mathrm{TiO}_{2}$ synthesized by aging method in low temperature was regular and spherical, to a certain extent. The particle size distribution was wide, ranging from $6 \mathrm{~nm}$ to $19 \mathrm{~nm}$. The average particle size was roughly estimated as $12 \mathrm{~nm}$ from the obtained images. These images suggested that synthesis of $\mathrm{TiO}_{2}$ in our microemulsion system with aging process at low temperature can bring about nanoparticles with relatively small and uniform sizes. 


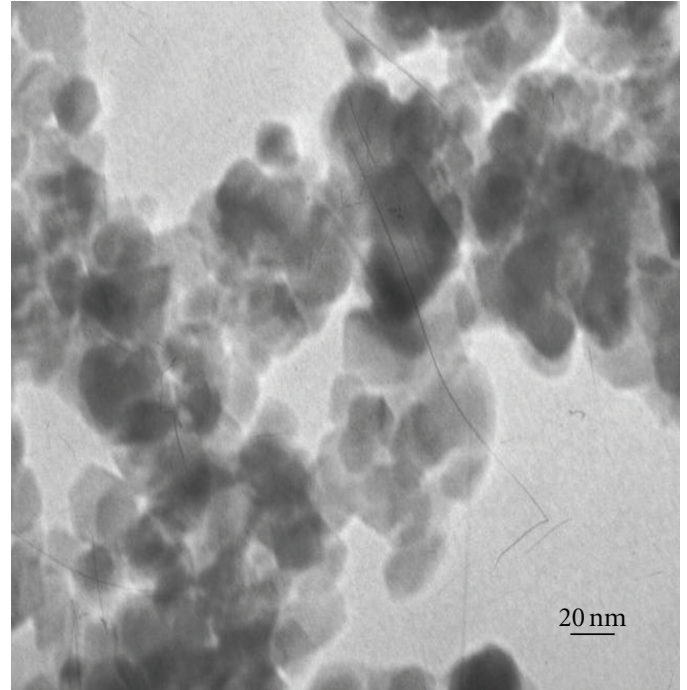

(a)

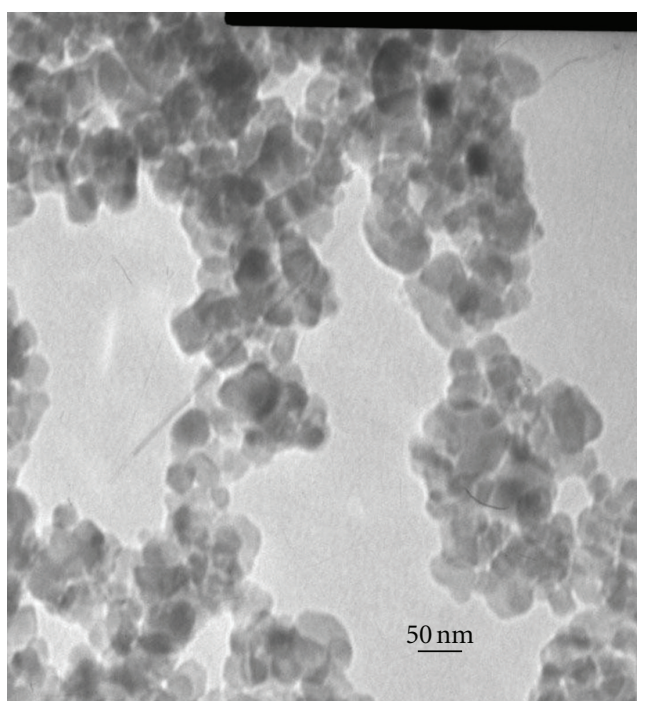

(b)

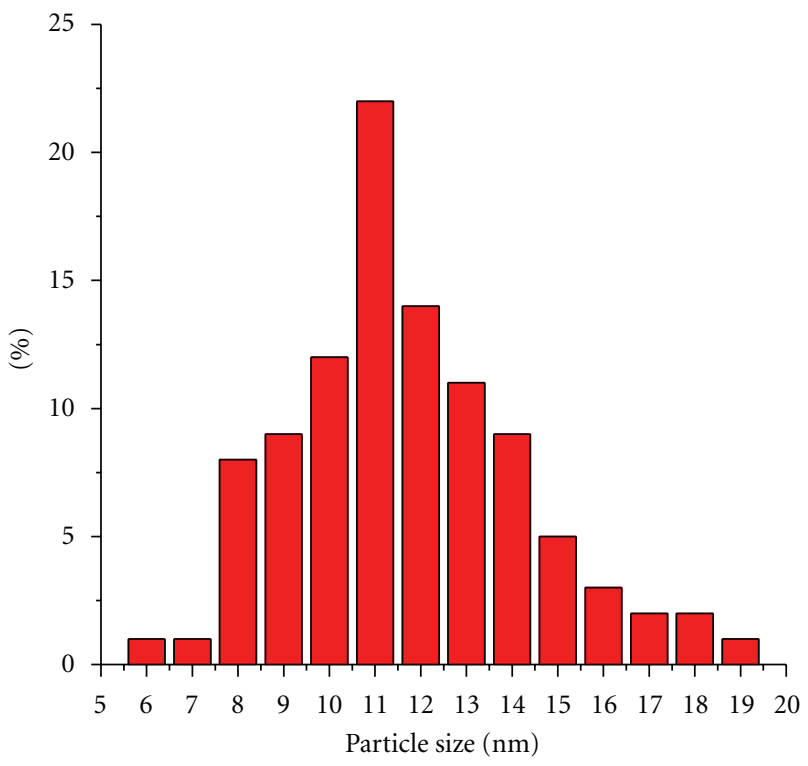

(c)

Figure 8: TEM and particle-size distribution graphs of $\mathrm{TiO}_{2}$ aging at $65^{\circ} \mathrm{C}$ for $120 \mathrm{~h}$.

3.3. Photocatalytic Activity. The degradation process of methyl orange (MO) was obtained by measuring the solution every 10 min during UV irradiation, and results were shown in Figure 9 as UV-Vis spectra curves. The photocatalytic reaction was under catalysis of $\mathrm{TiO}_{2}$ calcined at $500^{\circ} \mathrm{C}$ for $3 \mathrm{~h}$. There were two absorption peaks in the curves, the one at $464 \mathrm{~nm}$ was caused by the azo bond and the other one at $265 \mathrm{~nm}$ was caused by benzene ring [20]. During the decomposition process, both azo bond and benzene ring were degraded. In this research, the concentration of $\mathrm{MO}$ was roughly represented by the absorption at the maximum wavelength, which appeared at $464 \mathrm{~nm}$ as shown in Figure 9.

In order to compare the photocatalytic performance of as-obtained products synthesized in different conditions, various samples involving $\mathrm{TiO}_{2}$ calcined at $500^{\circ} \mathrm{C}$ for $3 \mathrm{~h}$,
$\mathrm{TiO}_{2}$ aged at $65^{\circ} \mathrm{C}$ for $72,90,120 \mathrm{~h}$, and $\mathrm{P} 25$ were conducted in UV-irradiated MO solutions, and degradation rates of MO were showed in Figure 10. It was demonstrated that the sample of $\mathrm{TiO}_{2}$ calcined at $500^{\circ} \mathrm{C}$ for $3 \mathrm{~h}$ had the best photoactivity which degraded $98 \%$ after $60 \mathrm{~min}$ (Figure 10 (a)). Sample of $\mathrm{TiO}_{2}$ aged at $65^{\circ} \mathrm{C}$ for $90 \mathrm{~h}$ also presented a high photoactivity which degraded over $74 \%$ after $60 \mathrm{~min}$ (Figure 10 (b)). These two samples had better photoactivity than commercial P25, which degraded $71 \%$ after 60 min (Figure 10 (c)). The relatively high photocatalytic activity of $\mathrm{TiO}_{2}$ aged at $65^{\circ} \mathrm{C}$ for $90 \mathrm{~h}$ was suggested to be responsible for the exhibited crystallinity and the enhanced adsorption ability caused by presence of amorphous titania. The results also showed that aging time affected photocatalytic activities of $\mathrm{TiO}_{2}$ obviously (Figure 10 (b), (d), and (e)). The efficiency 


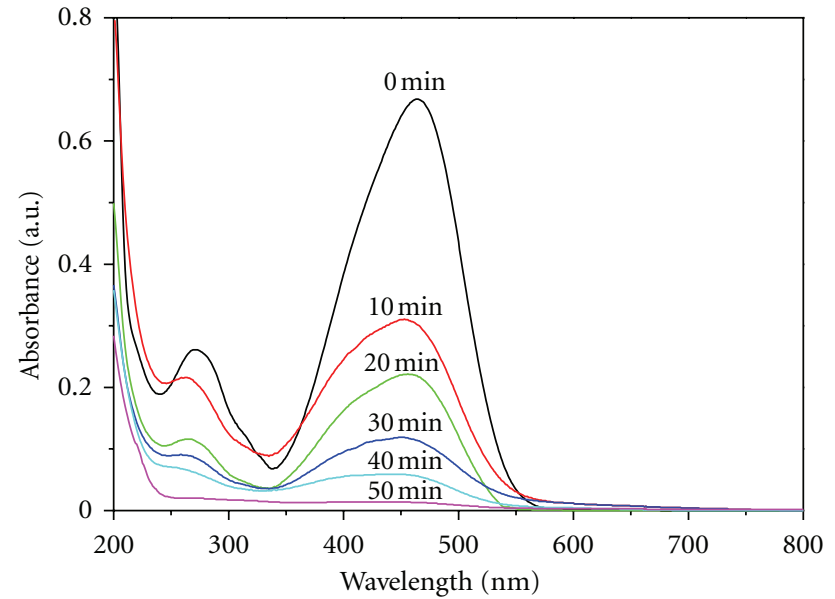

Figure 9: Degradation process of $\mathrm{MO}$ by aged $\mathrm{TiO}_{2}$ under ultraviolet light irradiation, analyzed by UV-Vis absorption spectra for solutions separated from the $\mathrm{TiO}_{2}$ suspensions irradiated for different periods.

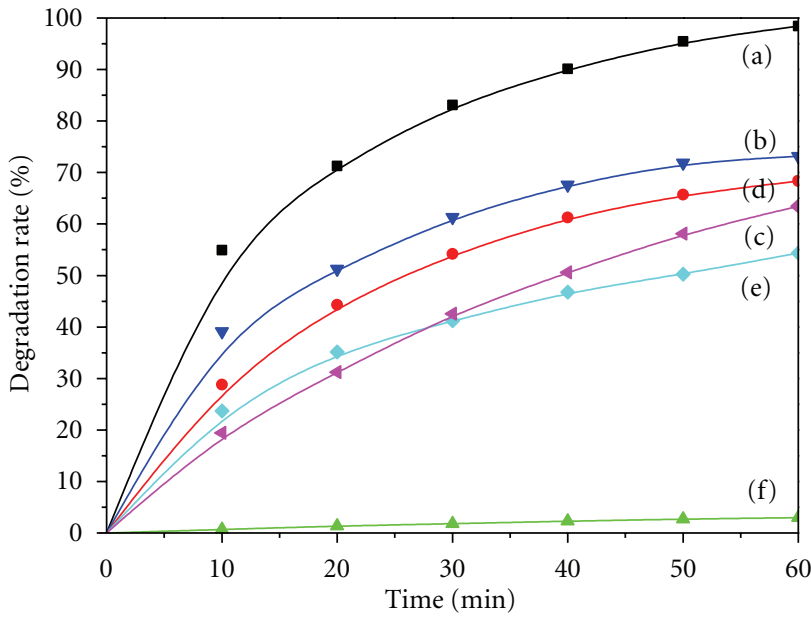

Figure 10: Degradation ratio of $\mathrm{MO}$ under ultraviolet light irradiation over catalysts as: (a) $\mathrm{TiO}_{2}$ calcined at $500^{\circ} \mathrm{C}$, (b) $\mathrm{TiO}_{2}$ aged at $65^{\circ} \mathrm{C}$ for $90 \mathrm{~h}$, (c) $\mathrm{TiO}_{2}$ aged at $65^{\circ} \mathrm{C}$ for $120 \mathrm{~h}$, (d) commercial P25, (e) $\mathrm{TiO}_{2}$ aged at $65^{\circ} \mathrm{C}$ for $72 \mathrm{~h}$, (f) blank.

of UV photocatalysis toward MO was low (Figure 10 (f)). $\mathrm{TiO}_{2}$ aged for $90 \mathrm{~h}$ represented the best photoactivity among samples prepared by aging process.

\section{Conclusions}

Nanosized $\mathrm{TiO}_{2}$ nanoparticles were synthesized in n-hexanol/CTAB/water reverse microemulsions. The precursors were treated at $500^{\circ} \mathrm{C}$ calcination or $65^{\circ} \mathrm{C}$ aging in water bath, and investigated by XRD, FT-IR, and TEM. The results show precursor is amorphous as $\mathrm{Ti}-\mathrm{Br}$ compounds; aging at low temperature is capable to form anatase $\mathrm{TiO}_{2}$ with reasonable photocatalytic performance. Among all the assynthesized photocatalysts by aging method, the nanoparticles aged at $65^{\circ} \mathrm{C}$ for $90 \mathrm{~h}$ showed the highest photocatalytic activity, and at the same time higher than that of commercial P25.

\section{Acknowledgment}

The authors would like to gratefully acknowledge the South China Normal University for financing this paper.

\section{References}

[1] J. A. Byrne, P. A. Fernandez-Ibañez, P. S. M. Dunlop, D. M. A. Alrousan, and J. W. J. Hamilton, "Photocatalytic enhancement for solar disinfection of water: a review," International Journal of Photoenergy, vol. 2011, Article ID 798051, 12 pages, 2011.

[2] J. Yu, J. C. Yu, M. K. P. Leung et al., "Effects of acidic and basic hydrolysis catalysts on the photocatalytic activity and microstructures of bimodal mesoporous titania," Journal of Catalysis, vol. 217, no. 1, pp. 69-78, 2003.

[3] Y. Fan, G. Chen, D. Li et al., "Highly selective deethylation of rhodamine B on $\mathrm{TiO}_{2}$ prepared in supercritical fluids," International Journal of Photoenergy, vol. 2012, Article ID 173865, 7 pages, 2012.

[4] P. Calandra, G. Calogero, A. Sinopoli, and P. G. Gucciardi, "Metal nanoparticles and carbon-based nanostructures as advanced materials for cathode application in dye-sensitized solar cells," International Journal of Photoenergy, vol. 2010, Article ID 109495, 15 pages, 2010.

[5] J. Yu, S. Liu, and H. Yu, "Microstructures and photoactivity of mesoporous anatase hollow microspheres fabricated by fluoride-mediated self-transformation," Journal of Catalysis, vol. 249, no. 1, pp. 59-66, 2007.

[6] H. Yu, J. Yu, S. Liu, and S. Mann, "Template-free hydrothermal synthesis of $\mathrm{CuO} / \mathrm{Cu}_{2} \mathrm{O}$ composite hollow microspheres," Chemistry of Materials, vol. 19, no. 17, pp. 4327-4334, 2007.

[7] Y. Lai, Y. Chen, H. Zhuang, and C. Lin, "A facile method for synthesis of $\mathrm{Ag} / \mathrm{TiO}_{2}$ nanostructures," Materials Letters, vol. 62, no. 21-22, pp. 3688-3690, 2008.

[8] C. H. Lu, W. H. Wu, and R. B. Kale, "Microemulsion-mediated hydrothermal synthesis of photocatalytic $\mathrm{TiO}_{2}$ powders," Journal of Hazardous Materials, vol. 154, no. 1-3, pp. 649-654, 2008.

[9] W. Liu and R. Guo, "The photostabilization of hemoglobin in triton $\mathrm{X}-100$ aqueous solution and triton $\mathrm{X}-100 / n-$ $\mathrm{C}_{5} \mathrm{H}_{11} \mathrm{OH} / \mathrm{H}_{2} \mathrm{O}$ microemulsion," Photochemistry and Photobiology, vol. 86, no. 4, pp. 835-843, 2010.

[10] C. Hu, S. Duo, T. Liu, J. Xiang, and M. Li, "Comparison of photocatalytic performance of anatase $\mathrm{TiO}_{2}$ prepared by low and high temperature route," Applied Surface Science, vol. 257, no. 8, pp. 3697-3701, 2011.

[11] L. Zhao, J. Ran, Z. Shu, G. Dai, P. Zhai, and S. Wang, "Effects of calcination temperatures on photocatalytic activity of ordered titanate nanoribbon $/ \mathrm{SnO}_{2}$ films fabricated during an EPD process," International Journal of Photoenergy, vol. 2012, Article ID 472958, 7 pages, 2012.

[12] N.-H. Lee, H.-J. Oh, S.-C. Jung, W.-J. Lee, D.-H. Kim, and S.-J. Kim, "Photocatalytic properties of nanotubular-shaped $\mathrm{TiO}_{2}$ powders with anatase phase obtained from titanate nanotube powder through various thermal treatments," International Journal of Photoenergy, vol. 2011, Article ID 327821, 7 pages, 2011.

[13] B. Qi, L. Wu, Y. Zhang, Q. Zeng, and J. Zhi, "Low-temperature and one-step synthesis of rutile $\mathrm{TiO}_{2}$ aqueous sol 
by heterogeneous nucleation method," Journal of Colloid and Interface Science, vol. 345, no. 2, pp. 181-186, 2010.

[14] A. R. Liu, S. M. Wang, Y. R. Zhao, and Z. Zheng, "Low-temperature preparation of nanocrystalline $\mathrm{TiO}_{2}$ photocatalyst with a very large specific surface area," Materials Chemistry and Physics, vol. 99, no. 1, pp. 131-134, 2006.

[15] A. Jada, J. Lang, and R. Zana, "Relation between electrical percolation and rate constant for exchange of material between droplets in water in oil microemulsions," The Journal of Physical Chemistry, vol. 93, no. 1, pp. 10-12, 1989.

[16] R. Duro, C. Souto, J. L. Gómez-Amoza, R. Martínez-Pacheco, and A. Concheiro, "Interfacial adsorption of polymers and surfactants: implications for the properties of disperse systems of pharmaceutical interest," Drug Development and Industrial Pharmacy, vol. 25, no. 7, pp. 817-829, 1999.

[17] D. Shechtman, I. Blech, D. Gratias, and J. W. Cahn, "Metallic phase with long-range orientational order and no translational symmetry," Physical Review Letters, vol. 53, no. 20, pp. 19511953, 1984.

[18] K. Lv, H. Zuo, J. Sun et al., "(Bi, C and N) codoped $\mathrm{TiO}_{2}$ nanoparticles," Journal of Hazardous Materials, vol. 161, no. 1, pp. 396-401, 2009.

[19] J. Fang, F. Wang, K. Qian, H. Bao, Z. Jiang, and W. Huang, "Bifunctional $\mathrm{N}$-doped mesoporous $\mathrm{TiO}_{2}$ photocatalysts," The Journal of Physical Chemistry C, vol. 112, no. 46, pp. 1815018156, 2008.

[20] S. Al-Qaradawi and S. R. Salman, "Photocatalytic degradation of methyl orange as a model compound," Journal of Photochemistry and Photobiology A, vol. 148, no. 1-3, pp. 161-168, 2002. 


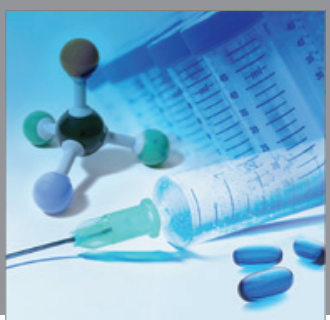

International Journal of

Medicinal Chemistry

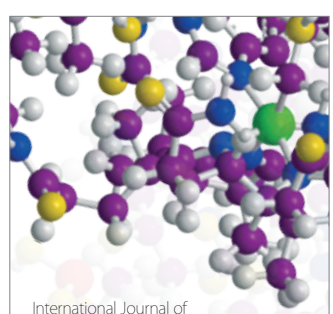

Carbohydrate Chemistry

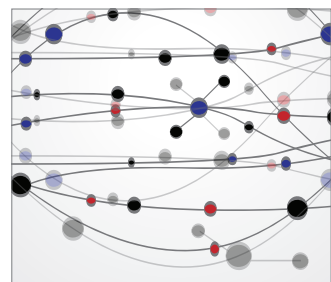

The Scientific World Journal
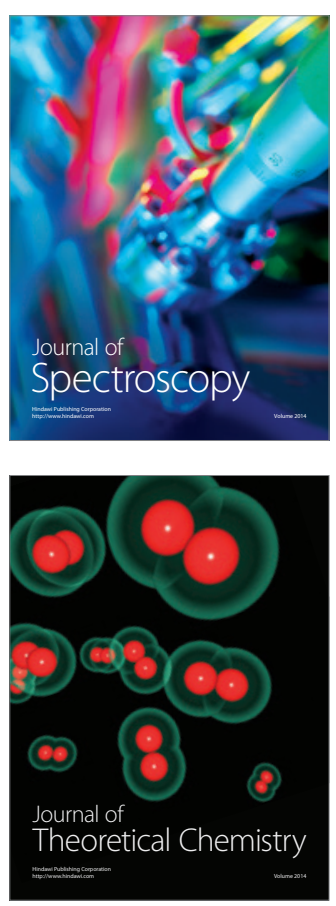
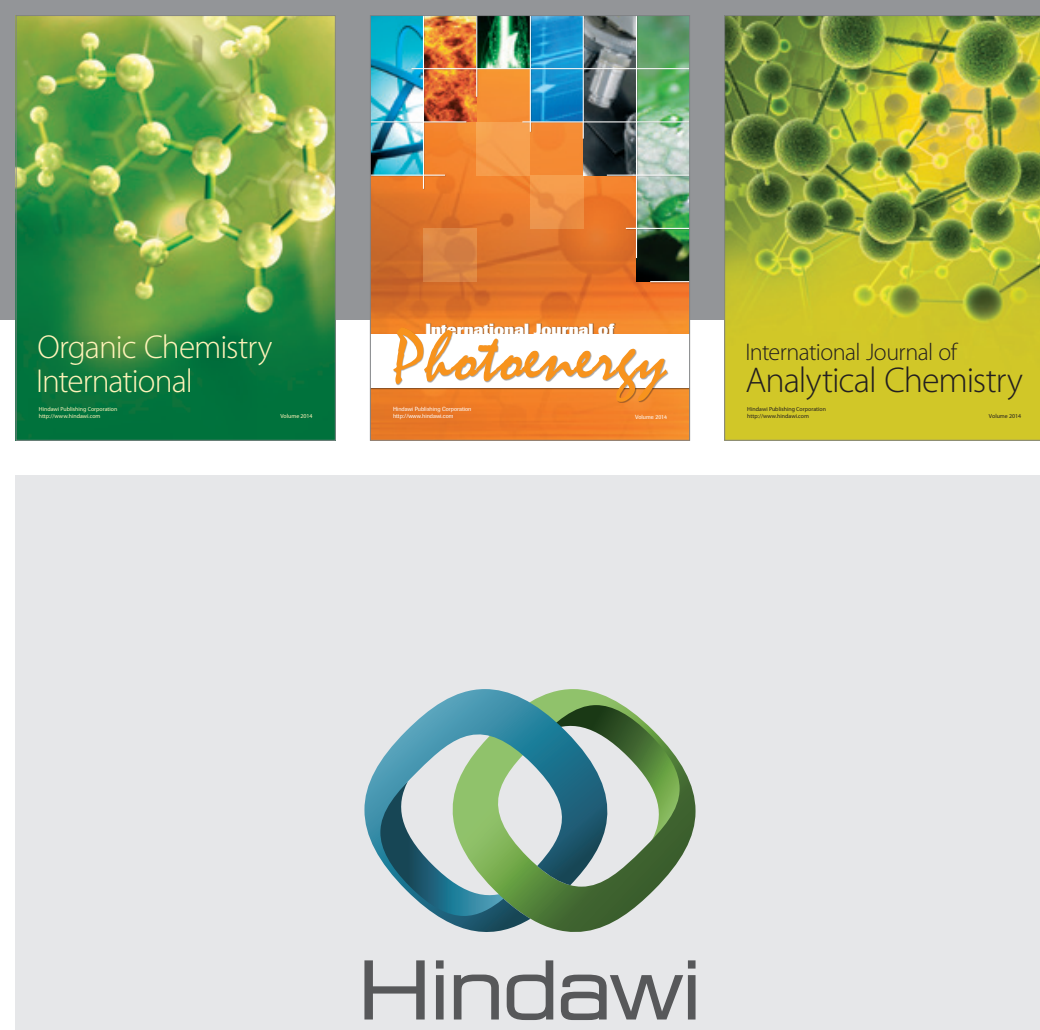

Submit your manuscripts at

http://www.hindawi.com
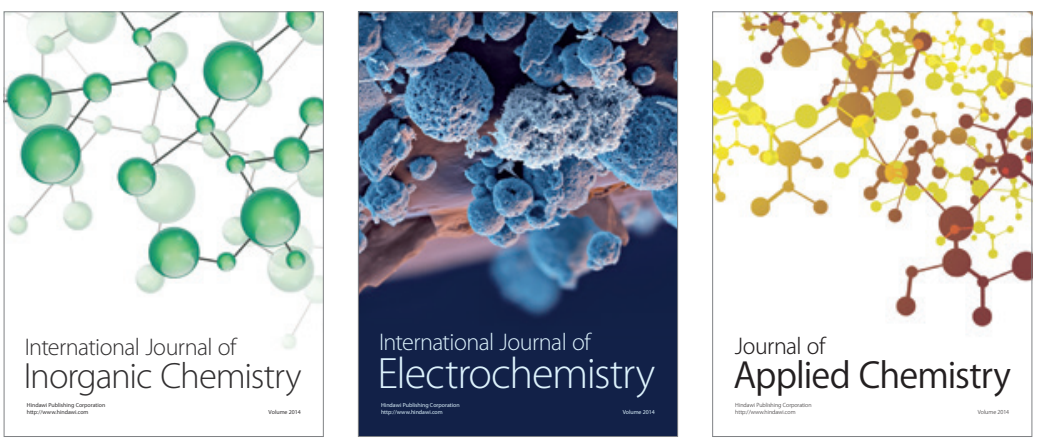

Journal of

Applied Chemistry
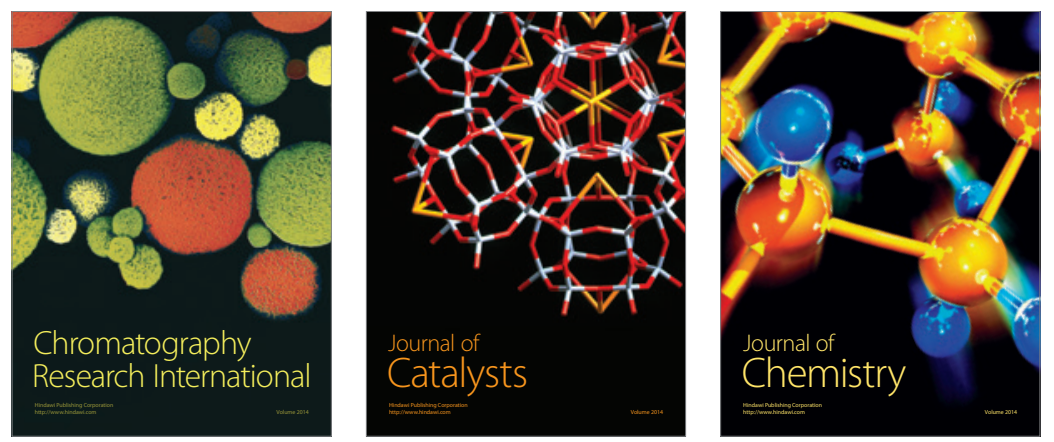
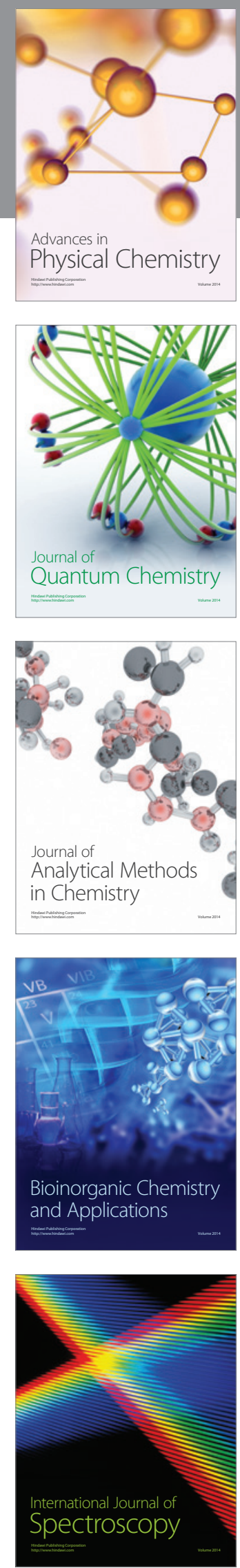